\title{
El Minuto de Dios, una mirada desde las ciencias sociales ${ }^{3}$
}

\author{
El Minuto de Dios, a look from the contemporary social sciences
}

\section{Resumen}

Para comprender el concepto de cambio social de Rafael García Herreros concretado en la Obra Social El Minuto de Dios, fundada en 1957, se hace una mirada desde las ciencias sociales contemporáneas, ayudados con algunos planteamientos teóricos de Talcott Parsons, Anthony Giddens y Amartya Sen. La base de este artículo de reflexión ha sido el análisis de contenido, ${ }^{4}$ realizado en el trabajo de grado de la maestría en antropología de Schuster R., H., (2006), De la Intervención a la Interacción Social. Una aproximación al contexto y cambios en la fundación de la obra social El minuto de Dios. Bogotá: Universidad de los Andes, Maestría en Antropología, limitándonos a analizar el concepto de cambio social en la obra de García Herreros.

Palabras clave: Minuto de Dios, García Herreros.

\begin{abstract}
To understand the concept of Social Change by Rafael Garcia Herreros, carried out in the social work at El Minuto de Dios, founded in 1957, it is made a look from the contemporary social sciences, aided with some theoretical approaches of Talcott Parsons, Anthony Giddens and Amartya Sen.

The basis of this reflective article has been the content's analysis developed at the Anthropology's masters degree work written by R. Schuster, H., (2006) "From Intervention to Social Interaction". An approach to the context and changes in the foundation of the social work at El Minuto de Dios, Bogotá Andes University, Masters in Anthropology, limiting ourselves to analyze the concept of social change in the work of Garcia Herreros.
\end{abstract}

Keywords: Minuto de Dios, García Herreros.

Recibido el 6 de noviembre de 2013 y aprobado el 12 de noviembre de 2013

1 Magíster en Teología de la Pontificia Universidad Gregoriana y Magíster en Antropología de la Universidad de los Andes. Investigador del Centro de Pensamiento Humano y Social - Uniminuto. Correo electrónico: hschustero@gmail.com.

2 Licenciado en Teología Seminario Mayor Nuestra Sra. de Suyapa (Honduras), estudiante de Maestría en Ciencia, Tecnología y Sociedad en la Universidad de Quilmes (Argentina). Tutor Uniminuto Virtual y Distancia. Correo electrónico: Igrubiano@gmail.com.

3 Artículo resultado de la investigación "De la Intervención a la Interacción Social. Una aproximación al contexto y cambios en la fundación de la obra social El minuto de Dios"

4 El material básico fue la lectura de algunos tomos de la obra completa, utilizando el Análisis de Contenido de Klaus Krippendorff, para remontarme a las ideas del fundador y tratar de evidenciar la intencionalidad de la fundación de la obra social. También se realizaron algunas entrevistas a algunos antiguos habitantes del barrio Minuto de Dios. 


\section{"El proyecto no es tener a los pobres irredentos, sino abrirles de par en par las puertas de un gran adelanto social sin paternalismo de ninguna clase"}

\author{
Rafael García Herreros (1961)
}

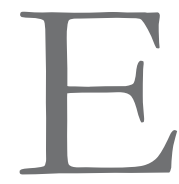

1 Minuto de Dios, una obra de carácter social cuya propuesta de desarrollo integral de las comunidades ha sido modelo de gestión para Colombia y el mundo, debe su origen a la iniciativa del sacerdote eudista Rafael García Herreros, quien en 1950 nombró así un corto espacio radial en el que reflexionaba sobre Dios, el hombre y el compromiso del pueblo cristiano.

Con la llegada de la televisión a Colombia, el programa diario pasó a la pantalla chica en 1955. En 1961 el padre decidió convocar el primer Banquete del Millón, con el objetivo de recoger fondos para construir soluciones de vivienda para los más pobres del país.

De estas iniciativas surgieron tanto el barrio El Minuto de Dios, modelo arquetípico de ciudadela integral como, a la postre, la Corporación El Minuto de Dios, dedicada a la construcción de comunidades y a la atención de poblaciones vulnerables en toda Colombia.

Tanto el programa de televisión, que el 10 de enero de 2008 cumplió 53 años de emisiones ininterrumpidas, como el Banquete del Millón (uno anual desde 1961) y el barrio que lleva el mismo nombre, subsisten hoy como parte de la obra viva de este visionario nacido en la ciudad de Cúcuta, en Colombia.

Otras instituciones de la organización El Minuto de Dios ofrecen servicios de educación básica y superior, crédito a la pequeña y mediana empresa, asesoría agroindustrial, comunicaciones y crecimiento espiritual para lograr elevar el nivel de vida de las gentes y propiciar su desarrollo humano integral.

\section{Gestión de El Minuto de Dios} en 55 años de servicio

Más de 53.000 casas entregadas a familias vulnerables; 26.100 familias asistidas a través de programas sociales; más de 640 niños beneficiados con el Plan Padrinos; 100.000 familias atendidas en situación de desplazamiento en los últimos 10 años; 4.578 personas beneficiadas en programas de Generación de Ingresos; nueve centros educativos; siete hogares infantiles (en convenio con el ICBF); 9.300 niños y jóvenes atendidos en nuestros centros educativos: Universidad con más de 80.000 jóvenes que estudian en las diferentes sedes del país; 136 programas con registro calificado; 4 emisoras; 20 librerías; más de 500 publicaciones propias; 78.000 personas capacitadas en las escuelas de evangelización.

El presente artículo es un estudio del concepto de cambio social en la fundación y primeros desarrollos de la obra social El Minuto de Dios, en la visión original de Rafael García Herreros, evidenciando el valor agregado que Parsons (1966, 1968), Giddens (1993) y Sen $(2000,2012)$ ofrecen a la comprensión del mismo.

Dicha mirada ofrece lineamientos a los gestores de cambio social de un modelo exitoso y plenamente vigente que ha sido producto de todo el desarrollo, praxis y saber-hacer de la obra social Minuto de Dios por más de 55 años de acción social en Colombia.

Este análisis suma a los trabajos que han abordado el tema de cambio social desde la perspectiva praxeológica de Juliao (2007 y 2011), desde las relaciones en la construcción de tejido social de Téllez (2009), desde el enfoque de la economía solidaria de Arias (2010) y desde las dinámicas comunitarias de Reyes (2010), los cuales abordan el tema de modo tangencial. Nuestro análisis trata directamente dicho tema y lo enriquece con miradas teóricas del pensamiento social contemporáneo en aras de una mayor comprensión de la obra de Rafael García Herreros, de sus objetivos y de su impacto. 
Dentro de las ciencias sociales contemporáneas existen diversos matices para abordar el tema del cambio social, los trabajos enunciados problematizan teóricamente la práctica de Rafael García Herreros, sin embargo falta hacer una reflexión más profunda respecto al tema del cambio social y este artículo pretende contribuir al tema, como artículo de reflexión.

\section{Materiales y métodos}

La base fundamental de este trabajo es el análisis de contenido realizado en la tesis de Maestría de Schuster (2006). Con este insumo se tomó la categoría de análisis cambio social, luego se hizo un barrido del tema en algunos textos de García Herreros que lo abordan directamente; se identificó el concepto en ellos y, por último, se caracterizó dicho tema. ${ }^{5}$ Hecho esto, se contrastó con las teorías de Parsons, Giddens y Sen para hallar elementos de comprensión del planteamiento de García Herreros en la fundación de la Obra Minuto de Dios.

Se escogieron estos autores, Parsons, Giddens y Sen, por la afinidad de sus propuestas teóricas del cambio social con la de García Herreros. Parsons habla de funcionalismo estructural, Giddens de la teoría de la estructuración y Sen de la teoría de la agencia, que son el equivalente al protagonismo que Rafael García Herreros le quería dar a los habitantes del barrio Minuto de Dios.

Estos tres autores escogidos son de relevancia en las ciencias sociales por sus aportes a las mismas. Talcott Parsons, sociólogo estadouni- dense perteneciente al paradigma estructuralfuncionalista, uno de sus principales objetivos a lo largo de su carrera académica fue el de crear un marco teórico de la acción unificada en ciencias sociales. Si bien no es considerado el fundador de ningún paradigma, a Parsons se le puede considerar como parte de la vertiente sociológica del funcionalismo, canonizado en la escuela británica de Antropología por figuras como Bronislaw Malinowski o Alfred Reginald Radcliffe-Brown. Anthony Giddens, sociólogo británico, ha contribuido en los más diversos campos de las ciencias sociales; ha reflexionado en torno a las más diversas figuras y escuelas de pensamiento, sirviéndose de los aportes teóricos tanto de la micro, como de la macrosociología. Amartya Sen, filósofo y economista bengalí (India), galardonado con el Premio Nobel de Economía en 1998; se le conoce por sus trabajos sobre las hambrunas, la teoría del desarrollo humano, la economía del bienestar y los mecanismos subyacentes de la pobreza.

Se analizaron varios tomos de la obra completa de Rafael García Herreros con la ayuda de Atlas.ti ${ }^{6}$ y las categorías de análisis fueron: vivienda, construcción de comunidad, espiritualidad, educación, economía solidaria, cultura, desarrollo, paz y reconciliación; se hizo un barrido con la categoría cambio social y otras afines, como adelanto social, desarrollo social, se hallaron las expresiones cambio social, adelanto social, desarrollo social; se caracterizaron, es decir, se realizó la inferencia del contexto en el cual estaban las expresiones para identificar el concepto de cambio social de García Herreros con todas sus características y detalles, y poderlo luego contrastar con las propuestas teóricas de Parsons, Giddens y Sen.

5 A esta técnica enunciada se le denomina "análisis de contenido", que se caracteriza por investigar el significado simbólico de los mensajes, los que no tienen un único significado, puesto que según menciona el autor: "Ios mensajes y las comunicaciones simbólicas tratan, en general, de fenómenos distintos de aquellos que son directamente observados" (Krippendorff, 1980).

6 Es un programa de computadora utilizado sobre todo, pero no exclusivamente, en la investigación cualitativa o análisis de datos cualitativos; un prototipo de Atlas.ti fue desarrollado por Thomas Muhr en la Universidad Técnica de Berlín, en el contexto del proyecto Atlas (1989-1992). 


\section{Discusión y resultados}

A partir del análisis se encontró que el término cambio social en Rafael García Herreros tiene diversos componentes, que en una mirada amplia cubren diversos campos presentados cronológicamente, que son también detonantes y evidencia de la realización del cambio social y de un camino consciente de construcción de un modelo replicable y propuesta suya para el pais, ${ }^{7}$ que fue construyendo e ideando inteligentemente.

Se presentan a continuación, y teniendo como punto de referencia la cronología, los campos de intervención de la obra social.

Vivienda (1957) ${ }^{8}$ : Ésta constituyó el tema básico de la obra social, se trataba "no del techo sino del vivir colectivo, es inmensamente complejo y requiere un sincero esfuerzo por hallarle solución" (García Herreros, 1961)․ La construcción de cambio social tiene en la posesión de vivienda los procesos alrededor de ésta y el compromiso familiar-comunitario que implica un dinamizador, que, junto a los demás elementos que estamos enunciando, logran cambio social real. Es importante anotar que el principal indicador de cambio social para Rafael García Herreros es el desarrollo integral, ${ }^{10}$ el cual tiene como objetivo la dignificación del ser humano como persona, comunidad y especie.
Construcción de comunidad $^{11}$ (1958): Fue la finalidad principal de la obra social, en relación esencial con la vivienda; ésta última es su estrategia de origen, pretexto-aliciente de la construcción de tejido social en los asentamientos humanos que realizó el Minuto de Dios durante más de 55 años. ${ }^{12}$ Este campo de acción es el punto de partida y llegada, junto con la dignificación del ser humano, de toda la acción social que Rafael García Herreros desarrolló. Un periodista le preguntó si era comunista, el padre contestó: "No soy comunista, soy comunitarista, sistema que pretende que la acción común, el esfuerzo común, la caridad común, la inteligencia común se apliquen en un momento dado en favor del individuo que requiere ayuda" (Jaramillo, 2009, p. 291).

El comunitarismo filosófico considera que el liberalismo clásico es ontológicamente y epistemológicamente incoherente, y se enfrenta al mismo en dos terrenos. A diferencia del liberalismo clásico, que construye a las comunidades como originadas por actos voluntarios de individuos anteriores a las mismas, remarca el papel de la comunidad en la tarea de definir y formar a los individuos. El comunitarismo ideológico subraya el derecho de la mayoría a tomar decisiones que afecten a la minoría. Se considera de izquierda en los asuntos económicos y de derecha en lo social. Una tercera posibilidad es el comunitarismo sensible (Responsive Communitarianism) que practica Amitai

7 "Lo que estamos tratando de realizar en Bogotá en el barrio Minuto de Dios, con la ayuda de muchos colombianos y como una experiencia que se puede multiplicar" (García Herreros, 1961).

8 Las fechas indican el orden cronológico de la aparición de las categorías.

9 "Quizá la vivienda no sea la necesidad fundamental que experimenta un hombre, pero poseerla puede ser causa de su despegue hacia el desarrollo: el hombre que logra adquirir casa propia se estimula para superarse en otros aspectos del progreso: presentación personal, higiene, salud, educación" (Jaramillo, 2009, p. 151).

10 Por desarrollo integral se entiende aquí: "la promoción de todos los hombres y de todo el hombre" y "el paso para cada uno y para todos de condiciones menos humanas a condiciones más humanas" (Pablo VI, 1967, pp. 20-21).

11 "Nuestra vivienda allá tiene estas características: es comunitaria. La estructura social evangélica lo es. Así lo entendieron los primeros cristianos. El cristianismo es el cuerpo místico de Cristo, es la más perfecta comunidad" (García Herreros, 1961).

12 "Los domingos hay obligación de trabajo común desde las 7 am. hasta las 9 am. Esta es la expresión más profunda de caridad y de solidaridad. Después de la conferencia para los hombres viene la santa misa, la máxima expresión comunitaria" (García Herreros, 1961). 
Etzioni (2001), quien propone una nueva regla de oro, para afirmar simultáneamente los derechos y obligaciones tanto comunitarios como individuales: "Respeta y defiende el orden moral de la sociedad de la misma manera que harías que la sociedad respetara y defendiera tu autonomía" (Martínez, 2005, p. 20). Aunque el término comunitarismo tiene su origen en el siglo XX, se puede encontrar elementos de esta filosofía en pensadores anteriores, como Robert Bellah, Charles Taylor, Michael Walzer y Alasdair MacIntyre.

Espiritualidad (1958): Constituida desde tres referentes: el amor-caridad, ${ }^{13}$ como fundamento y poder del cambio social; la pobreza, como aliciente ${ }^{14} \mathrm{y}_{\text {actitud }}{ }^{15}$; y por último, la experiencia personal y comunitaria de Dios trinidad ${ }^{16}$, como ambiente vital que, en reconocimiento existencial del valor de lo metafísico y trascendente, hace posible realizar un desarrollo integral y la dignificación de lo humano, de las personas y de las comunidades. Claramente para Rafael García Herreros y la realización de su propues- ta de cambio social es insuficiente con construir tejido social entre los seres humanos, se requiere también hacer comunión-tejido social con las tres personas divinas: Padre, Hijo y Espíritu Santo ${ }^{17}$ en una visión teológica.

Educación $^{18}$ (1957): Recién creado el barrio El Minuto de Dios, una de las decisiones del fundador fue abrir en 1957 una escuela que fue el germen de la Corporación Educativa Minuto de Dios, que educa actualmente a varios miles de alumnos. En la propuesta de Rafael García Herreros es otro de los campos fundamentales del cambio social (Juliao, 2007), ya que permite estructurar a los jóvenes y posibilitarles el paso a la educación superior $^{19}$. De hecho, muchos de los primeros egresados del Colegio Minuto de Dios (1967) pasaron a la Universidad Nacional. Tener una universidad en El Minuto de Dios era un sueño acariciado por el padre García Herreros durante muchos años (Jaramillo, 2009, p. 178). En algunos planos del barrio, elaborados en 1962, se indican unos predios para la futura y posible universidad ${ }^{20}$.

13 "El gran cambio social que se necesita no lo puede hacer el comunismo, que rechaza la fe y no conoce el amor. Somos los cristianos los que podemos y debemos realizarlo. El amor profundo lo transformaría todo. Cuando reine el amor, no habrá tugurios ni ignorancia ni hambre ni odios. Cuando reine el amor cristiano no habrá explotados, no habrá latifundios inútiles ni despilfarros" (Jaramillo, 2009, p. 291).

14 "¿Qué lo ha movido a ejecutar una obra tan gigantesca? "El convencimiento de que la miseria no es cristiana" (El Siglo, domingo 19 de marzo de 1961, p. 15).

15 "La pobreza beatificada en el Evangelio no es la carencia de lo necesario, sino la libertad interior del hombre ante las cosas. Carecer de lo necesario no es ser pobre según el Evangelio. Ser pobre según las bienaventuranzas es ser libre ante las riquezas, ante el mundo" (Jaramillo, 2009, p. 291).

16 "Ven, Espíritu Santo, me envuelvo en ti, quiero ser penetrado por ti, quiero ser conducido y transformado por ti; quiero difundir, quiero darle la Buena Nueva a muchos, de que tú consuelas al hombre, de que tú nos amas y nos enseñas a amar, y de que tú nos conduces hacia la infinita realidad de la Santísima Trinidad" (Jaramillo, 2009, p. 259).

17 "Qué bueno sentirnos hijos de un Padre infinito. Qué bueno saber que no estamos abandonados en un mundo hostil, sino que estamos cubiertos y protegidos por un amor paternal, que cuida todos los destinos y todos los pasos de nuestra vida" (García Herreros, 2008, p. 87).

18 "La educación es un bien que a todos beneficia y el camino para abolir la desigualdad entre los hombres" (Jaramillo, 2009, p. 169).

19 El 13 de noviembre del mismo año (1966), en La República, el periodista Libardo S. Tirado escribe, de seguro como eco a sus conversaciones con el padre García Herreros: "En El Minuto de Dios se proyecta la creación de una universidad tecnológica, donde los alumnos del barrio puedan, cuando menos, cursar dos años de educación superior y seguir posteriormente en otros centros universitarios del país o del exterior" (Jaramillo, 2009, p. 178).

20 "Diez años después, el 12 de julio de 1977, aparece esta nota en el libro de actas de la Junta Directiva de la Corporación El Minuto de Dios: Se trata el punto de la universidad en el barrio, que es un vivo anhelo del padre García Herreros. Se discute 
Economía solidaria (1959): La práctica de cambo social del Padre Rafael estuvo llena de iniciativas de economía solidaria que, surgidas de las necesidades de presente, se constituían en formas de apalancar las experiencias que se iban consolidando. Para buscar mejores rendimientos para la comunidad, se inició en 1959 una cooperativa de consumo asesorada, en sus comienzos, por Chrisnna Daryanani, un hindú; luego se organizó el fondo de empleados de la Corporación y finalmente la "Asociación Comunitaria de los habitantes del Minuto de Dios", entidad atenta a colaborar con las familias en caso de dificultad, enfermedad, muerte, estudios de los hijos, etc.

Estas iniciativas estaban siempre cargadas de gran dosis de realismo y orientadas a la autosostenibilidad, por ejemplo, en el reglamento inicial para los habitantes del Minuto de Dios el artículo 5 decía:

"Los comodatarios y su familia deben vincularse a la Cooperativa del Barrio "El Minuto de Dios", bien sea como afiliados a ella o bien adquiriendo por su conducto los artículos que vende, en iguales condiciones de calidad y precio a los que ofrecen otros expendios".

Cultura (1962) (Jaramillo, 2009, p. 201): Su visión del arte, expresión de la cultura y medio de formación integral, como un derecho a rescatar ${ }^{21}$ en la implementación del cambio social se plasma en el camino que llevó a constituir un museo de arte en el barrio El Minuto de Dios. A mediados de 1962 organizó un concurso de arte religioso, la idea se fue abriendo camino y en 1966 se inauguró el Museo. En un salón del colegio, en rústicos bastidores de guadua, se expusieron los primeros obsequios recibidos de los artistas que creyeron en la obra. Muchos otros artistas se han vinculado con la donación de obras: Fernando Botero, Alejandro Obregón, Eduardo Ramírez Villamizar, Omar Rayo, Edgar Negret, David Mazur, Augusto Rivera, Teresa Cuéllar, Pedro Alcántara, Enrique Grau, Armando Villegas, Rafael Penagos, Antonio Caballero, entre los colombianos, y Leonardo Nierman, José Luis Cuevas, Oswaldo Vigas, Justo Arosemena, entre los latinoamericanos, de modo que el fondo artístico supera actualmente las mil obras.

Desarrollo (1967): El sentido original fue el de formación integral que cambió cuando Rafael García Herreros asumió la definición de desarrollo de la encíclica de Pablo VI Populorum progressio que afirma que: "El verdadero desarrollo, que es el paso, para cada uno y para todos de condiciones de vida menos humanas, a condiciones más humanas" (1967, No. 20).

Paz y reconciliación ${ }^{22}$ (1985-1991): García Herreros propuso una forma de realizar el cambio social, pacífica, centrada en la agencia de los sujetos y generando sentido en las prácticas propuestas en la obra social por él fundada: "Yo sé que hay que hacer una revolución en favor tuyo, pero sin derramar una gota de sangre; una revolución con ciencia, con energía, con amor. Siento, hombre, la justicia de una revolución en tu favor" (Jaramillo, 2009, p. 233).

El cambio social: El quehacer de lo social de Rafael García Herreros, enunciado en los anteriores campos, fue construyendo una noción de cambio social que tiene las siguientes características:

la idea y se ve gran posibilidad de realizarla. Se piensa en un instituto técnico, a nivel intermedio, pero se propone hablar antes con las instituciones que pueden colaborar en su funcionamiento, como en las cuestiones técnicas a que haya lugar" (Jaramillo, 2009, p. 179).

21 "En realidad el problema no radicaba en si había en Colombia obras de gran valor artístico, sino en si la belleza tiene o no una función social" (Jaramillo, 2009, p. 165).

22 "Muchas páginas escritas por el padre García Herreros acerca del hombre, de sus derechos, de la solidaridad con los pobres, de la necesidad de construir un país nuevo, de la reconciliación y de la paz. Lo que caracteriza el pensamiento del padre Rafael y lo distingue de la teoría que pudiese exponer un sociólogo o un líder político es la inspiración de sus ideas: su propuesta brota del evangelio y de la doctrina social de la Iglesia" (Jaramillo, 2009, p. 261). 
1. Efectuar adelanto social autogestionado y fundado en el empoderamiento del sujeto de la acción social.

2. Eliminar todas las formas de miseria que aquejan a la persona y a las comunidades en su devenir humano, llamado a la realización plena y solidaria ${ }^{23}$.

3. Acceso irrestricto al derecho a vivir bien ${ }^{24}$, que todo ser humano tiene como inherente a su condición de hijo de Dios.

4. Construir una convivencia basada en la justicia, la reconciliación, la fraternidad y la $\mathrm{paz}^{25}$, desde una perspectiva sistémica y donde la interdependencia de estos valores construye la comunidad.

Otro elemento fundamental en la visión de Rafael García Herreros sobre el cambio social se encuentra en los principios que lo sustentan, en su práctica no son sólo elemento de referencia sino que se constituyen también en estrategia de eficacia y sostenibilidad, estos son: bien común, destino universal de los bienes, subsidiaridad, participación, solidaridad, verdad, libertad, justicia y amor. ${ }^{26}$
Expuesto lo anterior, abordemos ahora las posiciones teóricas de Parsons, Giddens y Sen que se constituyen en aporte para comprender la Obra Minuto de Dios, sus objetivos e impacto social.

El desarrollo reciente de la teoría social en relación al cambio social plantea una discusión sobre dos aspectos del mismo: los sujetos que intervienen en la realización del cambio y el modo de ejecutar ese cambio social (práctica social), para esto, en afinidad con la praxis del Rafael García Herreros, se propone pasar de los actores, que desempeñan un rol, ${ }^{27}$ a la agencia de los sujetos, ${ }^{28}$ donde hay participación, iniciativa, proactividad, protagonismo, autonomía. Otro tanto sucede con el ejercicio de la práctica social, que propone una dinámica de pasar de la acción simple, donde se privilegia el acto de la intervención, a la interacción de sujetos y procesos, centrada en el desarrollo de estrategias integradoras y el acompañamiento activo de procesos.

Referencia de lo anterior es la escuela estructural-funcionalista; sus orígenes se remontan a autores como Durkheim, quien da comienzo a la escuela funcionalista, y es perfeccionada

23 En 1961 un periodista le preguntó a Rafael García Herreros: "¿Qué lo ha movido a ejecutar una obra tan gigantesca? El convencimiento de que la miseria no es cristiana" (El Siglo, domingo 19 de marzo de 1961, p. 15). Esta fue su gran motivación. La inspiración y las herramientas las encontró en la Doctrina Social de la Iglesia, cuyos principios están presentes en la obra social (Pontificio Consejo Justicia y Paz, 2005).

24 "El logro más importante de esta época, no ha sido el invento de la fuerza atómica, ni la conquista del espacio cósmico, sino el despertar de la conciencia de que todos, sin excepciones, tenemos derecho a vivir bien, de que este derecho no es privativo de unos pocos. Para lograr realizarlo se necesita un cambio profundo" (García Herreros, 1961).

25 "El padre Rafael [...] nunca aceptó la violencia como solución, él rechazó la lucha de clases como método para construir un mundo nuevo, él siempre habló de un cambio profundo y de una revolución adelantada en paz, como consecuencia de saber que todo hombre es hijo de Dios y hermano de los otros hombres" (Jaramillo, 2009, p. 318).

26 "Lo que caracteriza el pensamiento del padre Rafael y lo distingue de la teoría que pudiese exponer un sociólogo o un líder político es la inspiración de sus ideas: su propuesta brota del evangelio y de la doctrina social de la Iglesia" (Jaramillo, 2009, p. 261).

27 Entendido como el papel que pone en práctica la persona, como el sistema de expectativas sociales que acompañan a la presentación pública de los sujetos de un determinado estado social o estatus.

28 Entendida como la posibilidad del sujeto de transformar el mundo. "Dicha teoría nace para dar satisfacción a algunos problemas no resueltos por otras y que se refieren a las relaciones que surgen dentro de la empresa y que se caracterizan, fundamentalmente, por la existencia de delegación de autoridad y coordinación de intereses contrapuestos entre los individuos" (Álvarez et al., 2000, p. 171). 
en el siglo XX por autores como Parsons y en Antropología por Radcliffe-Brown; para ellos la sociedad constituye un todo y para entender el equilibrio social, y en consecuencia el cambio social, es importante estudiar los componentes de la misma.

El estructural-funcionalismo plantea al ser humano en interacción con otros y sujeto de acciones que tienen determinantes de tipo social, cultural e institucional. Según la teoría estructural-funcionalista, la sociedad es un organismo y cada parte realiza una función. Todos los miembros de la sociedad concurren para cubrir sus necesidades porque tienen objetivos y valores en común.

El átomo de la teoría parsoniana es la acción, a la cual llama acto unidad. El acto unidad es una acción producida por un agente o actor. Su aporte a la interpretación de la acción social se evidencia al compararla con un sistema de interacción de los actores individuales que tienden a equilibrarse y cambiar paulatinamente. ${ }^{29}$

Parsons consideraba que la sociedad propende hacia la autorregulación y la autosuficiencia para satisfacer determinadas necesidades básicas, entre otras la preservación del orden social, el abastecimiento de bienes y servicios, y la protección de la infancia.

Al igual que en García Herreros, los radicalismos revolucionarios que plantean el cambio institucional total e inmediato no se dan dentro de la mirada de Parsons, sino que los cambios operan por reformas graduales que se introducen en el sistema y hacen que éstas evolucionen. Esta visión dominó las perspectivas dentro y fuera de las ciencias sociales, pero a partir de la primera mitad del siglo XX se fueron abriendo camino planteamientos antropológicos innovadores, destacadamente el relativismo cultural de Bronisław Malinowski y otros autores de la moderna antropología cultural (como Marvin Harris). Desde este tipo de mirada se comprende la desconfianza de García Herreros hacia los cambios radicales que planteaban los movimientos revolucionarios y de las teologías progresistas o de liberación. El cambio social se concibe en Parsons como una interdependencia entre actores-instituciones que gradualmente operan cambios cuando actores, en sus interacciones y acciones, ejercen presión a través de sistemas de mediaciones o procedimientos; dicho método fue precisamente aplicado por Rafael García Herreros con el sistema de alianzas interinstitucionales y de personas de altos ingresos, que fue construyendo para darle viabilidad y sostenibilidad a sus proyectos sociales (Trujillo et al., 2003).

En contraste con Parsons y en directa relación con el modo de proceder de García Herreros, Giddens (1998) establece que la acción social, los recursos y los conocimientos técnicos asociados a las prácticas de los agentes están determinados por procesos sociales que modifican los contextos y las estructuras sociales. En su visión alternativa, establece como categoría de comprensión del cambio social la relación agente-estructura, de recíproca interacción y con mayores márgenes para los agentes: éstos hacen historia, la modifican, la producen y reproducen. Esta perspectiva está en franca oposición con el estructural-funcionalismo parsoniano, donde los agentes son asimilados por la estructura y posee ésta mayor poder que aquellos.

Giddens, con su teoría de la estructuración, ${ }^{30}$ describe la producción y reproducción de la

29 "El punto fundamental es el concepto de los sistemas sociales de acción. En este sentido, la interacción de los actores individuales tiene lugar en condiciones tales que es posible considerar ese proceso de interacción como un sistema (en el sentido científico) y someterlo al mismo orden de análisis teórico que ha sido aplicado con éxito a otros tipos de sistemas en otras ciencias" (Parsons, 1966).

30 Para Giddens, "la estructura es "un "orden virtual" de relaciones transformadoras significa que los sistemas sociales, como prácticas sociales reproducidas, no tienen estructuras, sino más bien exhiben "propiedades estructurales", y que la estructura sólo existe como presencia espacio-temporal" (1998). 
sociedad por parte de los agentes sociales y no comparte la visión positivista teleológica o evolucionista de los proyectos sociales con pretensiones universales hegemónicas, propias de la Modernidad. Al igual que García Herreros, reconoce en las capacidades de los agentes los elementos definitivos que configuran la historia, mediante prácticas sociales transformadoras: ellas interfieren, alteran, producen y reproducen la sociedad.

Hay una apuesta decidida de Giddens por los agentes, contraria a la posición sistémica de Parsons. Los agentes sociales poseen estrategias subjetivas y racionalidades complejas que los positivistas descuidan o desconocen porque suponen que los procesos sociales son iguales en el tiempo y en el espacio. El agente tiene poder porque es capaz de modificar su entorno y su historia; tiene control sobre sus actos, sobre sí, a diferencia de los positivistas estructural-funcionalistas quienes ubican el control y lo determinante, exógeno al agente.

Para esta escuela, el orden, a semejanza de la naturaleza, es referencial y básico para la estructura social, lo cual no supone que Giddens desconozca las regularidades y los órdenes sociales, pero esto no agota el espacio de acción de los agentes, quienes, a través de las prácticas sociales, producen discontinuidades y multiplicidad de procesos sociales, rompiendo con las estandarizaciones y uniformidades del funcionalismo, produciendo diversidad histórica mediante sus prácticas; las estructuras son el instrumento y el resultado de esas prácticas sociales, elemento muy afín a la práctica social del padre Rafael, la cual era esencialmente de creación e innovación y renovación de estructuras (el barrio Minuto de Dios, el modelo de desarrollo y cambio social, el Banquete del Millón, los colegios, la Universidad).
Sin embargo, en la relación agente-estructura hay una dialéctica relacional de mutuo control que impide el determinismo estructural del positivismo y la libertad absoluta de los sujetos en las ciencias sociales. En otros términos, los agentes buscan el cómo, que supone recursos y técnica de conocimiento en el ejercicio de su práctica, delimitada en ambientes institucionales, con los márgenes de acción limitados de los agentes, éstos, a través de estrategia y recursos técnicos de conocimiento, amplian o maximizan el margen de maniobra y poder de sus prácticas y acciones sociales, modificando el entorno para el logro de sus fines específicos e históricos: los agentes reproducen estructuras por sus prácticas y las circunstancias las tematizan. Para la teoría de la estructuración de Giddens (1993), los contextos, acontecimientos, situaciones históricas y las prácticas sociales están sujetas a cambios que producen variabilidad histórica, contenidos específicos y contextos, lo que nos permite comprender la práctica social de García Herreros como realizada en la dialéctica de los elementos mencionados por Giddens y nos abre un camino adicional de análisis de su obra y método de cambio social.

En cuanto al rol del agente de cambio, Sen coloca el término en relación simbiótica con el de "agencia social", de este modo despoja el término agente de un sesgo individualizante y ligado al ejercicio de liderazgo, a una visión más democrática, participativa y solidaria. Agente es cualquier persona: "que actúa y provoca cambios y cuyo logros pueden juzgarse en función de su propios valores y objetivos." ${ }^{31}$ De este modo avala la intuición práctica de García Herreros donde los procesos de cambio no los realiza centrado en líderes y liderazgos, sino que se dan en el empoderamiento de personas y equipos para realizar agencia social, en la amplia participación en la construcción de la Obra Minuto de Dios y en

31 "Aquí no utilizamos el término "agente" en este sentido, sino en el más antiguo y "elevado" de la persona que actúa y provoca cambios y cuyos logros pueden juzgarse en función de sus propios valores y objetivos, independientemente de que los evaluemos o no también en función de algunos criterios externos [...] al papel de la agencia del individuo como miembro de lo público y como participante en actividades económicas, sociales y políticas (que van desde participar en el mercado hasta intervenir directa o indirectamente en actividades individuales o conjuntas en el terreno político y de otros tipos)" (Sen, 2000, pp. 35-36). 
el desarrollo de la capacidad individual y comunitaria para lograr cambios concretos y visibles en la realidad.

En la perspectiva de Sen, la persona es agente porque ejerce agencia social (2002, pp. 35-36), se reconoce como actor fundamental de los procesos de cambio y, por tanto, se entiende como responsable de actuar para incidir en ellos. Como en Rafael García Herreros, el momento primero no es hacer agentes para construir el cambio y el momento segundo es la ejecución del cambio, en contraposición. García Herreros hace del realizar el cambio un proceso pedagógico de conversión del individuo hacia la agencia social y al entenderse como agente de cambio, en tanto va construyendo desde su actual identidad un cambio visible, va modificando también su percepción de sí mismo y orientando su ser e identidad como agente efectivo de cambio. ${ }^{32}$

\section{Conclusiones}

Una de las características que García Herreros a los destinatarios y beneficiarios de la obra, involucrándolos en procesos de desarrollo humano y social sostenibles ${ }^{34}$, elementos fundamentales del cambio social; buscó el concurso de la gente, una verdadera interacción social, formando a las personas para realizar agencia social, para el cambio social que involucrara a los sujetos, sus acciones y elecciones, en los ambientes y contextos definidos y diferenciados con los que los sujetos lidian en sus prácticas sociales.

Se evidencia en la práctica social ejemplificada en la obra de García Herreros un desplazamiento similar al reseñado en la teoría social del sujeto como actor cumpliendo roles a la de la agencia contemporánea del sujeto, de los proyectos de intervención a favor de los sectores sociales a la búsqueda de mecanismos de autogestión, autoayuda, participación e interacción.

Entre los elementos que potencian la agencia se encuentran la participación, la responsabilidad y la educación, que constituye la expansión de la libertad y las oportunidades, elementos que fortaleció García Herreros en El Minuto de Dios ${ }^{35}$, haciendo que los destinatarios de su acción, más que pacientes fueran agentes de su propio desarrollo, sostenibilidad y cambio social. obra fue la de tratar de empoderar ${ }^{33}$, se diría hoy,

32 "[...] de concebir a los individuos, incluso a los beneficiarios, como agentes [...] activos de cambio [...] más que como pacientes inmóviles [...] Comprender el papel de la agencia es, pues, fundamental para reconocer que las personas son personas responsables: [...] actuamos o nos negamos a actuar y podemos decidir actuar de una forma u otra" (Sen, 2000, pp. 233-234).

33 Proceso por el cual las personas fortalecen sus capacidades, confianza, visión y protagonismo como grupo social para impulsar cambios positivos de las situaciones que viven. La filosofía del empoderamiento tiene su origen en el enfoque de la educación popular desarrollada a partir del trabajo en los años sesenta de Paulo Freire, estando ambas muy ligadas a los denominados enfoques participativos, presentes en el campo del desarrollo desde los años 70. Recuperado de http://www.dicc.hegoa. ehu. es/listar/mostrar/86

34 "Para que el hombre logre una superación constante se requiere que participe libremente en los procesos de transformación y que cuente con la solidaridad de quienes lo rodean" (Jaramillo, 2004). "Aquí no utilizamos el término "agente" en este sentido, sino en el más antiguo y "elevado" de la persona que actúa y provoca cambios y cuyos logros pueden juzgarse en función de sus propios valores y objetivos, independientemente de que los evaluemos o no también en función de algunos criterios externos. [...] al papel de la agencia del individuo como miembro de lo público y como participante en actividades económicas, sociales y políticas (que van desde participar en el mercado hasta intervenir directa o indirectamente en actividades individuales o conjuntas en el terreno político y de otros tipos)" (Sen, 2000, pp. 35-36).

35 "Por eso, desde sus primeros días, cuando apenas había un puñado de casas en los extramuros de Bogotá, adoptó unos reglamentos que marcarían pautas de conducta a todos los habitantes de su barrio: aseo, educación, trabajo, participación en las actividades comunes" (Jaramillo, 2004). 
Expresado en su lenguaje el agente social, del que nos habla Sen, aparece como alternativa de participación de la comunidad, como una respuesta colectiva a la cultura de la insolidaridad, es un impulsor de iniciativas sociales, de barrios, de zonas deprimidas. Es un dinamizador de sectores sociales con problemas. Es una persona que, desde una conciencia solidaria, comprende que no lleva soluciones, sino que comparte con los demás la búsqueda de las mismas. El agente social se involucra en un proceso de cambio estructural parsoniano y de construcción de dinámicas alternativas que se definen desde la solidaridad. Expresa su solidaridad social a través del acompañamiento social necesario para un proceso de autonomía y participación de las comunidades para que logren el ejercicio de los derechos sociales.

La propuesta de García Herreros de articular y movilizar los distintos actores y sectores sociales de Colombia, en función de proyectos colectivos que transformasen la sociedad y las instituciones, por la participación activa y colectiva de los ciudadanos va en sintonía con la percepción de autores como Giddens en cuanto al protagonismo de los agentes para definir, construir y modificar sociedad a través de procesos y prácticas sociales colaborativas y participativas.

Los primeros esfuerzos sociales de Rafael García Herreros se encaminaron a adjudicar viviendas a familias numerosas de escasos recur$\operatorname{sos}^{36}$ Algunas familias beneficiadas quisieron aprovechar la vivienda sin ofrecer ninguna reciprocidad a la obra naciente, esto ayudó a afinar el objetivo de El Minuto de Dios que no podía reducirse sólo a construir casas, sino que debía ampliarse a la formación de comunidades (García Herreros, 1961) organizadas social, económica y gremialmente, en una constante superación (Reyes Escobar, 2010).
El Minuto de Dios, desde su creación hasta hoy, ha pretendido estimular a las personas y a las comunidades para que sean conscientes de su dignidad de seres humanos, de sus derechos, de la realidad en la que viven, y de la participación y solidaridad necesarias para su constante superación. Esa toma de conciencia entendida como un proceso de humanización, mediante el que los hombres se dan cuenta de su ser y de sus circunstancias y pasen de una actitud pasiva, de actores que desempeñan un rol, a un dinamismo transformador, agentes del cambio social, que requiere un análisis crítico, que descubra lo que es injusto e inaceptable, que encuentre posibilidades para llevar a cabo el cambio, que despliegue energías y ponga en marcha procesos para ir de lo menos humano a lo más humano.

Para que el hombre logre una superación constante se requiere que participe libremente en los procesos de transformación y que cuente con la solidaridad de quienes lo rodean. Distintos documentos eclesiales orientan esta concepción. Pablo VI enseñó que: "el desarrollo es el nuevo nombre de la paz" (1967, No. 87) y Juan Pablo II afirma que: "la paz es fruto de la solidaridad" (2001). Por lo tanto, si somos solidarios, contribuiremos al desarrollo y cosecharemos paz.

Podemos concluir que Parsons, Giddens y Sen desde sus miradas teóricas ofrecen elementos de comprensión de los objetivos, el impacto de la obra:

Muy convencido de su opción escogió un camino medio, guiado por el pensamiento social que elaboró a lo largo de los años, primero de manera teórica, marcado por sus estudios sociales en Europa y por la atenta lectura del magisterio social de la Iglesia disponible hasta la década del cincuenta, y luego enriquecido por la praxis social, que lo abrió en su pensamiento. Escogió

36 "Las casas se entregaban en obra negra y sin servicios públicos. Para ser favorecido se necesitaban tres requisitos: familia de bajos recursos económicos, más de cinco hijos y ser católicos" (García, 2009). 
una vía caracterizada por la independencia y autonomía, lo que le valió no sucumbir ante las tentaciones de los radicalismos y extremismos propios de la época, ya que ese era el Zeitgeist ${ }^{37}$ que le correspondió vivir y en el que se enmarca la fundación de la obra social. Conoció de cerca esas experiencias y a sus protagonistas con los cuales tuvo afinidades y diferencias, pero finalmente escogió un camino propio, una praxis social particular.

\section{Referencias}

Álvarez P., et al. (abril, 2000). Las cooperativas en el marco de la teoría de la agencia en Ciriec-España. Revista de Economía Pública, Social y Cooperativa, 34, Recuperado de http:// www.ciriec-revistaeconomia.es/banco/07_ Alvarez_34.pdf

Arias G., J. (2010). Rafael Garcia-Herreros y el Minuto de Dios emprendimiento social para el siglo XXI en Colombia. 1909-2009, centenario de su natalicio.

El Siglo (1961). Entrevista al Padre Rafael García Herreros "¿Qué lo ha movido a ejecutar una obra tan gigantesca? "El convencimiento de que la miseria no es cristiana”. Domingo 19 de marzo de 1961 p. 15.

Etzioni, A. (2001). La tercera via hacia una buena sociedad: propuestas desde el comunitarismo. España: Trotta.

García Herreros, R. (1961). Conferencia presentada en la Universidad de Antioquia. Archivo histórico Minuto de Dios.
- (2009). Artesanos de Paz.

Colección Obras Completas No.18. Bogotá: Corporación Centro Carismático Minuto de Dios.

. (2009). Constructores de la nueva Colombia. Colección Obras Completas No.19. Bogotá: Corporación Centro Carismático Minuto de Dios. . (2010). Una Hora Para Dios. Colección Obras Completas No.20. Bogotá: Corporación Centro Carismático Minuto de Dios. . (2010). El Banquete del Millón. Colección Obras Completas No.21. Bogotá: Corporación Centro Carismático Minuto de Dios.

. (2011). Palabras del Pastor Vol.1. Colección Obras Completas No.22. Bogotá: Corporación Centro Carismático Minuto de Dios.

37 Es originalmente una expresión del idioma alemán que significa "el espíritu (Geist) del tiempo (Zeit)". Muestra el clima intelectual y cultural de una era. 
. (2011). Palabras del Pastor Vol.2. Colección Obras Completas No.23. Bogotá: Corporación Centro Carismático Minuto de Dios.

. (2011). Palabras del Pastor Vol.3. Colección Obras Completas. Bogotá: Corporación Centro Carismático Minuto de Dios.

(2011). Palabras del Pastor Vol.4. Colección Obras Completas. Bogotá: Corporación Centro Carismático Minuto de Dios.

Giddens, A. (1993). Las Nuevas Reglas del Método Sociológico. Buenos Aires: Amorrortu.

- (1998). La constitución de la sociedad. Bases para la teoría de la estructuración. Buenos Aires: Amorrortu.

Gómez C., J. (2007). Renovación Carismática Católica (RCC) en Bogotá. Una propuesta de sentido en la segunda mitad del siglo XX (Tesis de Maestría) Universidad de los Andes, Bogotá.

Jaramillo, D. (2004). Rafael García Herreros: una vida y una obra. Bogotá: Centro Carismático el Minuto de Dios.

Juan Pablo II. (2001, 28 de marzo). Discurso a la delegación de una editora austriaca.

Juliao, C. (2006). El educador social como gestor de comunidades autogestionarias. Praxis Pedagógica, 6, 80-89.

. (2007). El Minuto de Dios: una opción por el Desarrollo humano y social y un modelo de gestión y educación social. En O. Useche Aldana, C. M. Naranjo Plata, \& L. M. Cabrera Ardila (Eds.). Desarrollo, ciuda- dania y cambio social. Desafíos para las politicas públicas en Colombia (pp. 347-352). Bogotá: Uniminuto.

. (2007). Educación Social. El

Minuto de Dios: una experiencia y un modelo. Bogotá: Corporación Universitaria Minuto de Dios, Facultad de Educación y Centro de Estudios e Investigaciones Humanas y Sociales, CEIHS.

Krippendorff, K. (1980). Metodología de análisis de contenido: teoría y práctica. Barcelona: Paidós.

Martínez J.B. (2005). Educación para la ciudadanía. Madrid: Morata.

Pablo VI. (1967). Populorum progressio, Carta encíclica sobre "el desarrollo de los pueblos".

Parsons, T. (1966). El sistema social. Madrid: Revista de Occidente.

- (1968). La estructura de la acción social: estudio de teoría social, con referencia a un grupo de recientes escritores europeos. Madrid: Guadarrama.

Pérez, H. (2007). Rafael García Herreros y el Minuto de Dios: contextos (1959-1968) (Tesis de maestría inédita) Universidad de los Andes, Bogotá.

Pontificio Consejo Justicia y Paz. (2005). Compendio de la Doctrina Social de la Iglesia. Ciudad del Vaticano: Libreria Editrice Vaticana.

Rocher, G. (1985). Introducción a la sociología general. Barcelona: Herder.

Reyes Escobar, N. C. (2010). Comunidady sentido de patria: discurso y práctica en el barrio Minuto de Dios 1956-1992. Bogotá: Uniminuto. 
Schuster R. H. (2006). De la Intervención a la Interacción Social. Una aproximación al contexto y cambios en la fundación de la obra social El minuto de Dios. (Tesis de Maestría). Universidad de los Andes, Bogotá.

(2008) Al Abrigo de un sueño. Utopía realizada. Bogotá: Uniminuto.
Sen, A. (2000). Desarrollo y Libertad. Bogotá: Planeta.

. (2012). La Idea de la Justicia Madrid: Santillana.

Téllez, E. (2009). Los Vínculos y el Barrio El Minuto de Dios. Polisemia 7 (1), 15-25. 